SCIENTIFIC LETTER

\title{
Transvenous low energy internal cardioversion for atrial fibrillation refractory to external cardioversion: do non- obese patients benefit?
}

\author{
D Pavin, H Legrand, C Leclercq, C Crocq, P Mabo, J C Daubert
}

Heart 2004;90:332-333. doi: 10.1136/hrt.2003.010082

t patients with persistent atrial fibrillation $(\mathrm{AF})$, restoration of sinus rhythm can be accomplished by external

electrical cardioversion (EEC) with a $70-90 \%$ success rate. The effectiveness of external cardioversion is linked to transthoracic impedance. Total body weight is a significant factor in determining transthoracic impedance and cardioversion failure is more frequent in obese patients. ${ }^{1}$ The technique of transvenous low energy internal cardioversion (LEIC) overcomes those limitations. ${ }^{2}$ It remains to be established if LEIC will be an effective therapeutic option in non-obese patients in which failure of external cardioversion may be caused by the underlying heart disease and long term sinus rhythm maintenance more problematic.

The aim of this prospective study was to assess the immediate and long term effectiveness of LEIC in a series of consecutive patients with persistent AF refractory to EEC, with special reference to the outcome in obese and non-obese patients.

\section{PATIENTS AND METHODS}

Consecutive patients with persistent AF ( $\geqslant 8$ days) refractory to transthoracic monophasic shocks (with at least two attempts at $360 \mathrm{~J}$ in an anteroposterior paddle position) were included. A cardioversion attempt was designated as unsuccessful if sinus rhythm was not restored for at least one beat. Exclusion criteria were: (1) hyperthyroidism; (2) acute and transient cause of AF; (3) decompensated heart failure at the time of attempted cardioversion; (4) cardiac surgery within three months.

Shocks were delivered using single twin-coil catheters: 6 French custom designed catheters (Tad Cath 8027/8029, In Control, Redmond, Washington, USA; and Vascostim, VascoMed, Weil am Rhein, Germany) in 53 patients and a 7 French catheter (Alert Catheter, EPMed Systems, Mt Arlington, New Jersey, USA) in six patients. Techniques for transvenous catheter insertion and cardioversion have been described in detail elsewhere. ${ }^{34}$ Biphasic (3/3 ms) shocks were delivered under light sedation (morphine chlorhydrate) using custom designed external atrial defibrillators, at energy levels up to $400 \mathrm{~V}$ or $15 \mathrm{~J}$. Surface and intracardiac ECG were continuously recorded during shock delivery and successful cardioversion was defined as the occurrence of at least one sinus rhythm beat.

All variables are expressed as percentage or mean (SD) value. Differences in categorical variables between groups were analysed using the $\chi^{2}$ test. Continuous variables were compared using the Student's $t$ test or Mann-Whitney U test, as appropriate. Estimates of the proportion of patients without AF recurrence as a function of time was established using the Kaplan-Meier method and the groups were compared according to the log rank test. Association between variables, initial outcome (successful cardioversion) and subsequent relapse into AF were explored by univariate and multivariate analysis (Wald and Cox logistic regression model). Significance was achieved at $\mathrm{p}<0.05$.

\section{RESULTS}

Fifty nine consecutive patients with AF refractory to EEC (50 men; age 62 (10) years) underwent internal cardioversion. Body weight was 91 (19) kg (57-140 kg). The median duration of the current AF episode was four months (eight days to 108 months). AF was idiopathic in 18 patients $(31 \%)$ and associated with cardiovascular heart disease in 41 patients $(69 \%)$, mainly hypertension $(n=25)$. Left ventricular ejection fraction (LVEF) was $0.48(0.11)$ and left atrial diameter was 49 (8) $\mathrm{mm}$.

Sinus rhythm was restored by LEIC in 45 patients $(76 \%)$ with a mean energy of 7.3 (3.1) J. There were no significant differences between the patients in whom internal cardioversion was successful or unsuccessful regarding sex, age, weight, duration of AF, structural heart disease, LVEF or left atrial diameter.

The mean follow up duration of the 45 patients whose sinus rhythm was restored by LEIC was 18 (3) months (2-34 months). Antiarrhythmic drugs prescribed after cardioversion (physician's preference) included amiodarone in 23 patients $(51 \%)$, flecainide in 9 patients $(20 \%)$, sotalol in 8 patients $(18 \%)$, and no treatment in 5 patients $(11 \%)$. AF recurred in 23 patients $(51 \%)$. The proportion of patients without AF recurrence was 55 (7)\% after one year, $49(8) \%$ after two years, and 39 (11)\% after three years. No predictors of subsequent $\mathrm{AF}$ recurrence could be determined in a multivariate analysis of clinical (sex, age, weight, duration of AF, New York Heart Association functional class, structural heart disease, amiodarone treatment) or echocardiographic variables (LVEF, left atrial diameter).

Of the 59 patients included in the study, 35 (59\%) exhibited obesity as defined by a body mass index $\geqslant 30 \mathrm{~kg} /$ $\mathrm{m}^{2}$. There were no significant differences in clinical or echocardiographic variables between obese and non-obese patients. Sinus rhythm was restored by internal shocks in 29 of the 35 obese patients ( $83 \%$ ) versus 16 of the 24 non-obese patients $(67 \%)(p=0.15)$. The risk of AF recurrence after sinus rhythm restoration was the same in obese and nonobese patients (fig l).

\section{DISCUSSION}

Successful cardioversion depends on the nature of the underlying heart disease and the current density delivered to the atrial myocardium. The latter, in turn, depends on

Abbreviations: $A F$, atrial fibrillation; $E E C$, external electrical cardioversion; LEIC, low energy internal cardioversion; LVEF, left ventricular ejection fraction 


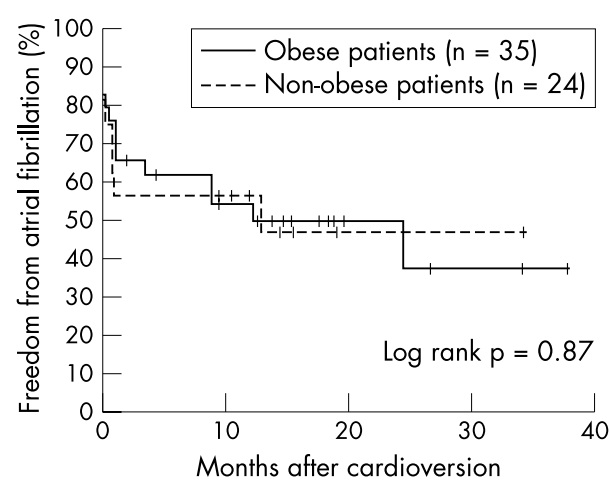

Figure 1 Kaplan-Meier plots showing the proportion of obese (body mass index $\geqslant 30 \mathrm{~kg} / \mathrm{m}^{2}$ ) and non-obese (body mass index $<30 \mathrm{~kg}$ / $\mathrm{m}^{2}$ ) patients remaining in sinus rhythm after successful internal cardioversion.

transthoracic impedance which is correlated to the increase in thoracic diameter and body size. Thus, body weight has been recognised as a factor of external shock failure. ${ }^{1}$ Obese patients are considered as good candidates for internal cardioversion because the failure of external cardioversion is merely linked to an "extrinsic" technical factor-that is, the low current density delivered to atrial tissue. Furthermore, it is generally thought that external cardioversion failure in non-obese patients is mainly related to "intrinsic" factors, which are more dependent on the electrophysiological substrate or underlying heart disease, as well as on episode duration or atrial size. Those factors should be associated with more AF recurrences provided sinus rhythm can be restored first.

In this study, we did not find any significant difference between obese and non-obese subjects admitted for internal cardioversion. If the immediate success of internal cardioversion appeared greater in obese patients $(83 \%)$, the difference with non-obese patients, whose sinus rhythm was restored in two thirds of cases, was not significant $(p=0.15)$. Lastly, we did not find any significant difference in AF recurrence risk between obese and non-obese patients. So, on the basis of our study, the effectiveness of internal cardioversion appears not to be closely related to body weight and therefore should not be reserved for obese people.

A monophasic shock waveform was used for external cardioversion in the study. Use of a biphasic waveform would likely necessitate internal cardioversion less frequently ${ }^{5}$ and the effectiveness of LEIC will undoubtedly need to be reassessed in such a context.

\section{Authors' affiliations}

D Pavin, H Legrand, C Leclercq, C Crocq, P Mabo, J C Daubert, Département de Cardiologie et Maladies Vasculaires, Centre Hospitalier, Universitaire, Rennes, France

Correspondence to: Dr D Pavin, Département de Cardiologie et Maladies Vasculaires, Centre Cardio-Pneumologique, Hôpital Pontchaillou, 2 rue Henri Le Guilloux, 35033 Rennes, France; dominique.pavin@chu-rennes.fr

Accepted 11 July 2003

\section{REFERENCES}

1 Lévy S, Lauribe P, Dolla $E$, et al. A randomised comparison of external and internal cardioversion of chronic atrial fibrillation. Circulation 1992:86:1415-20.

2 Boriani G, Biffi M, Camanini C, et al. Transvenous low energy internal cardioversion for atrial fibrillation: a review of clinical applications and future developments. PACE 2001;24:99-107.

3 Andraghetti A, Scalese M. Safety and efficacy of low-energy cardioversion of 500 patients using two different techniques. Europace $2001 ; 3: 4-9$.

4 Plewan A, Valina C, Herrmann R, et al. Initial experience with a new balloonguided single lead catheter for internal cardioversion of atrial fibrillation and dual chamber pacing. PACE 1999:22(Pt II):228-32.

5 Mittal S, Ayati S, Stein KM, et al. Transthoracic cardioversion of atrial fibrillation. Comparison of rectilinear biphasic versus damped sine wave monophasic shocks. Circulation 2000;101:1282-7. 